\title{
Stability of the $d$-wave pairing with respect to the intersite Coulomb repulsion in cuprate superconductors
}

\author{
V. V. Val'kov ${ }^{a}$, D. M. Dzebisashvili ${ }^{a}$, M. M. Korovushkin ${ }^{a}$, A.F. Barabanov ${ }^{b}$ \\ ${ }^{a}$ Kirensky Institute of Physics, Federal Research Center KSC SB RAS, 660036 Krasnoyarsk, Russia \\ ${ }^{b}$ Vereshchagin Institute for High Pressure Physics, 108840 Troitsk, Russia
}

\begin{abstract}
Within the spin-fermion model for cuprate superconductors, the influence of the intersite Coulomb interactions $V_{2}$ and $V_{2}^{\prime}$ between holes located at the next-nearest-neighbor oxygen ions of $\mathrm{CuO}_{2}$ plane on the implementation of the $d_{x^{2}-y^{2}}$-wave pairing is studied. It is shown that $d$-wave pairing can be suppressed only for unphysically large values of $V_{2}$ and $V_{2}^{\prime}$.
\end{abstract}

\section{INTRODUCTION}

It is known that the real structure of $\mathrm{CuO}_{2}$ plane is characterized by the spatial separation of the subsystem of holes located at oxygen ions and the subsystem of spins localized at copper ions (Fig. 11). Besides, a 'number of features is caused by the presence of two oxygen ions in the unit cell of copper-oxygen plane. The minimal realistic microscopic model for cuprates is the three-band $p-d$ model (the Emery model) [1,2. This model takes into account the $d_{x^{2}-y^{2} \text {-orbitals of copper }}$ ions and $p_{x^{-}}$and $p_{y}$-orbitals of oxygen ions. However, along with the realism, the multiband character of the 'Emery model leads to cumbersome analysis of cuprates physics. That is why a number of studies in this direction is carried out in the framework of the Hubbard model and its effective low-energy variants, such as $t-J$ and $t-J^{*}$ models on the simple square lattice. In these models, the same fermions form the charge and the spin subsystems.

Along with the number of important results, such an approach has a serious disadvantage: the Cooper 'pairing of fermions caused by the kinematic [3, ex'change 4, 5, and spin-fluctuation mechanisms considered in the Hubbard [6],7, $t-J$ [4,5], or $t-J^{*}$ [8,9] mod'els is suppressed by the intersite Coulomb repulsion $V_{1}$ of charge carriers located at the neighboring sites. This 'effect is most pronounced in the $d$ channel [10] and the Cooper instability disappears completely at $V_{1} \sim 1-2$ $\mathrm{eV}$.

In our previous paper 11, it has been shown that, because of the two-orbital character of the subsystem of holes located at oxygen sites and the spatial separation of this subsystem from that of spins at copper ions, the superconducting phase in high- $\mathrm{T}_{c}$ cuprates is stable with respect to the strong Coulomb repulsion of holes located at the nearest-neighbor oxygen sites if the order

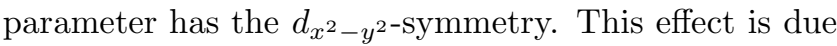
to the symmetry properties of the Coulomb potential.

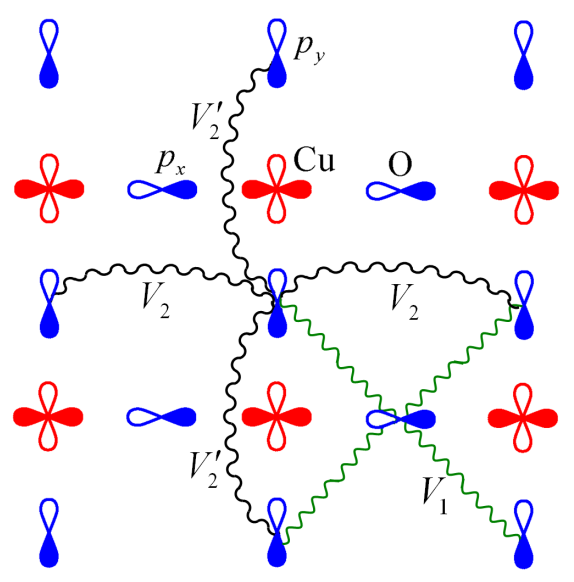

Fig. 1. Structure of $\mathrm{CuO}_{2}$ plane. Here $V_{1}$ denotes the Coulomb interaction between holes located at the nearest-neighbor oxygen sites and $V_{2}$ and $V_{2}^{\prime}$ correspond to the Coulomb interactions of holes located at the nextnearest-neighbor oxygen sites.

Note that in Ref. 11 the stability of the $d$-wave pairing was proved only for the case of the intersite Coulomb repulsion of holes located at the nearest-neighbor oxygen ions, $V_{1}$, while the role of the Coulomb repulsion between holes located at the more distant oxygen ions, $V_{2}$, is still unclear (the possibility of influence of $V_{2}$ on the superconducting $d$-wave pairing has been also mentioned in Ref. [12]). In this paper, we study the role of the Coulomb interaction between holes located at the next-nearest-neighbor oxygen ions on $\mathrm{CuO}_{2}$-plane in the implementation of the superconducting $d_{x^{2}-y^{2}}$ wave pairing.

\section{MODEL}

In the strongly correlated regime, when the Hubbard repulsion energy $U_{d}$ is large, i.e., $U_{d}>\Delta_{p d} \gg t_{p d}$, the $p-d$ model is reduced to the spin-fermion model [13, 14] 
describing the subsystem of oxygen holes interacting with the spins located at copper ions. The Hamiltonian of the spin-fermion model is represented in the form

$$
\begin{aligned}
\hat{H}= & \hat{H}_{0}+\hat{J}+\hat{I}+\hat{V} \\
\hat{H}_{0}= & \sum_{k \alpha}\left(\xi_{0}\left(k_{x}\right) a_{k \alpha}^{\dagger} a_{k \alpha}+\xi_{0}\left(k_{y}\right) b_{k \alpha}^{\dagger} b_{k \alpha}\right. \\
& \left.+t_{k}\left(a_{k \alpha}^{\dagger} b_{k \alpha}+b_{k \alpha}^{\dagger} a_{k \alpha}\right)\right), \\
\hat{J}= & \frac{J}{N} \sum_{f k q \alpha \beta} e^{i f(q-k)} u_{k \alpha}^{\dagger}\left(\mathbf{S}_{f} \boldsymbol{\sigma}_{\boldsymbol{\alpha} \boldsymbol{\beta}}\right) u_{q \beta}, \quad \hat{I}=\frac{I}{2} \sum_{\langle f m\rangle} \mathbf{S}_{f} \mathbf{S}_{m}, \\
\hat{V}= & V_{2} \sum_{f} \hat{n}_{f+\frac{x}{2}} \hat{n}_{f+\frac{x}{2}+y}+V_{2} \sum_{f} \hat{n}_{f+\frac{y}{2}} \hat{n}_{f+\frac{y}{2}+x} \\
& +V_{2}^{\prime} \sum_{f} \hat{n}_{f+\frac{x}{2}} \hat{n}_{f+\frac{x}{2}+x}+V_{2}^{\prime} \sum_{f} \hat{n}_{f+\frac{y}{2}} \hat{n}_{f+\frac{y}{2}+y},
\end{aligned}
$$

where

$$
\begin{aligned}
& \xi_{0}\left(k_{x(y)}\right)=\varepsilon_{p}-\mu+\tau\left(1-\cos k_{x(y)}\right), \\
& t_{k}=(2 \tau-4 t) \sin \frac{k_{x}}{2} \sin \frac{k_{y}}{2}, \\
& u_{k \beta}=\sin \frac{k_{x}}{2} a_{k \beta}+\sin \frac{k_{y}}{2} b_{k \beta}, \\
& \tau=\frac{t_{p d}^{2}}{\Delta_{p d}}\left(1-\frac{\Delta_{p d}}{U_{d}-\Delta_{p d}-2 V_{p d}}\right), \\
& J=\frac{4 t_{p d}^{2}}{\Delta_{p d}}\left(1+\frac{\Delta_{p d}}{U_{d}-\Delta_{p d}-2 V_{p d}}\right), \\
& I=\frac{4 t_{p d}^{4}}{\left(\Delta_{p d}+V_{p d}\right)^{2}}\left(\frac{1}{U_{d}}+\frac{2}{2 \Delta_{p d}+U_{p}}\right) .
\end{aligned}
$$

The Hamiltonian $\hat{H}_{0}$ describes the oxygen holes in the momentum representation. Here $a_{k \alpha}^{\dagger}\left(a_{k \alpha}\right)$ are the hole creation (annihilation) operators in the oxygen subsystem with the $p_{x}$-orbitals (Fig. 1), $\alpha= \pm 1 / 2$ is the spin projection. Similarly, $b_{k \alpha}^{\dagger}\left(b_{k \alpha}\right)$ are operators in the oxygen subsystem with the $p_{y}$-orbitals. The bare one-site energy of oxygen holes is $\varepsilon_{p}, \mu$ is the chemical potential, and $t$ is the hopping integral. The operator $\hat{J}$ describes the exchange interaction between the subsystem of oxygen holes and the subsystem of the spins localized at copper ions. Here, $\mathbf{S}_{f}$ is the operator of a spin localized at site with index $f$ and $\boldsymbol{\sigma}=\left(\sigma^{x}, \sigma^{y}, \sigma^{z}\right)$ is the vector of the Pauli matrices. The operator $\hat{I}$ describes the superexchange interaction between the neighboring spins at copper ions. The intersite Coulomb interaction between holes is described by the operator $\hat{V}$. As far as the role of the Coulomb repulsion $V_{1}$ between holes located at the nearest oxygen sites was clarified in Ref. 11], here we do not take into account the corresponding term in the Hamiltonian $\hat{V}$ and restrict ourselves to a treatment of the interactions $V_{2}$ and $V_{2}^{\prime}$ be- tween the next-nearest neighbors (Fig. (1). In the Hamiltonian, $\hat{n}_{f+x(y) / 2}=\sum_{\sigma} \hat{n}_{f+x(y) / 2, \sigma}$ is the operator of the number of holes at the oxygen site $f+x(y) / 2$, where $x=(1,0)$ and $y=(0,1)$ are the lattice basis vectors in the units of the lattice parameter.

When writing the Hamiltonian (1), we take into account that the hopping integrals in the first and the second terms can have different signs for different hopping directions owing to the different phases of the wave functions.

Below we use the commonly accepted set of parameters of the model: $t_{p d}=1.3 \mathrm{eV}, \Delta_{p d}=3.6 \mathrm{eV}$, $U_{d}=10.5 \mathrm{eV}, V_{p d}=1.2 \mathrm{eV}$ [15]17]. Note that for this set, the parameter of the superexchange energy $I=0.136 \mathrm{eV}(1570 \mathrm{~K})$ agrees well with the experimental data on cuprate superconductors 17. For the hopping integral of the holes, we use the value $t=0.1 \mathrm{eV}$ [18, and we suppose that the parameters of the intersite Coulomb interactions are $V_{2}=V_{2}^{\prime}=$ $0.5-1.5 \mathrm{eV}$.

It is important that the exchange energy between the localized and itinerant spins calculated using the expression (3) is large, namely, $J=3.4 \mathrm{eV} \gg \tau \approx 0.1 \mathrm{eV}$. Therefore, to describe the oxygen holes dynamics it is necessary to take into account the exchange interaction rigorously. This problem is solved using the following basis set of operators [18, 19]

$$
a_{k \alpha}, \quad b_{k \alpha}, \quad L_{k \alpha}=\frac{1}{N} \sum_{f q \beta} e^{i f(q-k)}\left(\mathbf{S}_{f} \boldsymbol{\sigma}_{\alpha \beta}\right) u_{q \beta},
$$

where the third operator describes the strong spincharge coupling.

\section{EQUATIONS FOR GREEN'S FUNCTIONS}

For consideration of the conditions for the Cooper instability, we supplement the basis set (4) by the operators $(\bar{\alpha}=-\alpha)$

$$
a_{-k \bar{\alpha}}^{\dagger}, \quad b_{-k \bar{\alpha}}^{\dagger}, \quad L_{-k \bar{\alpha}}^{\dagger} .
$$

The equations for the normal $G_{i j}$ and the anomalous $F_{i j}$ Green's functions obtained by the method [20,21] can be represented in the form $(j=1,2,3)$

$$
\begin{aligned}
& \left(\omega-\xi_{x}\right) G_{1 j}=\delta_{1 j}+t_{k} G_{2 j}+J_{x} G_{3 j}+\Delta_{1 k} F_{1 j}, \\
& \left(\omega-\xi_{y}\right) G_{2 j}=\delta_{2 j}+t_{k} G_{1 j}+J_{y} G_{3 j}+\Delta_{2 k} F_{2 j}, \\
& \left(\omega-\xi_{3}\right) G_{3 j}=\delta_{3 j} K_{k}+\left(J_{x} G_{1 j}+J_{y} G_{2 j}\right) K_{k}+\Delta_{3 k} F_{3 j}, \\
& \left(\omega+\xi_{x}\right) F_{1 j}=\Delta_{1 k}^{*} G_{1 j}-t_{k} F_{2 j}-J_{x} F_{3 j}, \\
& \left(\omega+\xi_{y}\right) F_{2 j}=\Delta_{2 k}^{*} G_{2 j}-t_{k} F_{1 j}-J_{y} F_{3 j}, \\
& \left(\omega+\xi_{L}\right) F_{3 j}=\Delta_{3 k}^{*} G_{3 j}-\left(J_{x} F_{1 j}+J_{y} F_{2 j}\right) K_{k} .
\end{aligned}
$$


Here, $G_{11}=\left\langle\left\langle a_{k \uparrow} \mid a_{k \uparrow}^{\dagger}\right\rangle\right\rangle, \quad G_{21}=\left\langle\left\langle b_{k \uparrow} \mid a_{k \uparrow}^{\dagger}\right\rangle\right\rangle$, and $G_{31}=\left\langle\left\langle L_{k \uparrow} \mid a_{k \uparrow}^{\dagger}\right\rangle\right\rangle$. The functions $G_{i 2}$ and $G_{i 3}$ are determined in a similar way with the only difference that $a_{k \uparrow}^{\dagger}$ is replaced by $b_{k \uparrow}^{\dagger}$ and $L_{k \uparrow}^{\dagger}$, respectively. The anomalous Green's functions are defined as $F_{11}=$ $\left\langle\left\langle a_{-k \downarrow}^{\dagger} \mid a_{k \uparrow}^{\dagger}\right\rangle\right\rangle, F_{21}=\left\langle\left\langle b_{-k \downarrow}^{\dagger} \mid a_{k \uparrow}^{\dagger}\right\rangle\right\rangle, F_{31}=\left\langle\left\langle L_{-k \downarrow}^{\dagger} \mid a_{k \uparrow}^{\dagger}\right\rangle\right\rangle$.

For $F_{i 2}$ and $F_{i 3}$, the same type of notation regarding the second index is used. The functions involved in (6) are determined by the expressions

$$
\begin{aligned}
& \xi_{x(y)}=\xi_{0}\left(k_{x(y)}\right), \quad J_{x(y)}=J \sin \frac{k_{x(y)}}{2}, \quad K_{k}=\frac{3}{4}-C_{1} \gamma_{1 k}, \\
& \xi_{L}(k)=\varepsilon_{p}-\mu-2 t+5 \tau / 2-J \\
& \quad+\left[(\tau-2 t)\left(-C_{1} \gamma_{1 k}+C_{2} \gamma_{2 k}\right)+\tau\left(-C_{1} \gamma_{1 k}+C_{3} \gamma_{3 k}\right) / 2\right. \\
& \left.\quad+J C_{1}\left(1+4 \gamma_{1 k}\right) / 4-I C_{1}\left(\gamma_{1 k}+4\right)\right] K_{k}^{-1} .
\end{aligned}
$$

Here, $\gamma_{j k}$ are the square lattice invariants: $\gamma_{1 k}=$ $\left(\cos k_{x}+\cos k_{y}\right) / 2, \quad \gamma_{2 k}=\cos k_{x} \cos k_{y}, \quad \gamma_{3 k}=$ $\left(\cos 2 k_{x}+\cos 2 k_{y}\right) / 2$. In the course of deriving ([6), we assume that the state of localized moments corresponds to the quantum spin liquid. Therefore, the spin correlation functions $C_{j}=\left\langle\mathbf{S}_{0} \mathbf{S}_{r_{j}}\right\rangle$ satisfy the relations

$$
C_{j}=3\left\langle S_{0}^{x} S_{r_{j}}^{x}\right\rangle=3\left\langle S_{0}^{y} S_{r_{j}}^{y}\right\rangle=3\left\langle S_{0}^{z} S_{r_{j}}^{z}\right\rangle,
$$

where $r_{j}$ is the position of a copper ion within the coordination sphere $j$. Besides, $\left\langle S_{f}^{x}\right\rangle=\left\langle S_{f}^{y}\right\rangle=\left\langle S_{f}^{z}\right\rangle=0$.

From (6), it follows that the spectrum of the Fermi excitations in the normal phase is determined by the solution of the dispersion equation

$$
\begin{aligned}
& \operatorname{det}_{k}(\omega)=\left(\omega-\xi_{x}\right)\left(\omega-\xi_{y}\right)\left(\omega-\xi_{L}\right)-2 J_{x} J_{y} t_{k} K_{k} \\
& -\left(\omega-\xi_{y}\right) J_{x}^{2} K_{k}-\left(\omega-\xi_{x}\right) J_{y}^{2} K_{k}-\left(\omega-\xi_{L}\right) t_{k}^{2}=0 .
\end{aligned}
$$

The spectrum is characterized by three bands $\epsilon_{1 k}, \epsilon_{2 k}$ and $\epsilon_{3 k}$ [22]. The branch $\epsilon_{1 k}$ with the minimum at a point close to $(\pi / 2, \pi / 2)$ of the Brillouin zone arises owing to the strong spin-fermion coupling. At the low value of the number of holes per one oxygen ion $n_{p}$, the dynamics of holes is determined by the characteristics of the lower band $\epsilon_{1 k}$. This band is separated by an appreciable gap from the upper bands $\epsilon_{2 k}$ and $\epsilon_{3 k}$ [22].

The introduced order parameters $\Delta_{j, k}$ are related to the anomalous averages as follows

$$
\begin{aligned}
\Delta_{1 k}=-\frac{2}{N} \sum_{q} & \left(V_{2} \cos \left(k_{y}-q_{y}\right)\right. \\
& \left.+V_{2}^{\prime} \cos \left(k_{x}-q_{x}\right)\right)\left\langle a_{q \uparrow} a_{-q \downarrow}\right\rangle, \\
\Delta_{2 k}=-\frac{2}{N} \sum_{q} & \left(V_{2} \cos \left(k_{x}-q_{x}\right)\right. \\
& \left.+V_{2}^{\prime} \cos \left(k_{y}-q_{y}\right)\right)\left\langle b_{q \uparrow} b_{-q \downarrow}\right\rangle,
\end{aligned}
$$

$$
\begin{aligned}
& \Delta_{3 k}=\frac{1}{N} \sum_{q} I_{k-q}\left\{\left\langle L_{q \uparrow} L_{-q \downarrow}\right\rangle+C_{1 x}\left\langle a_{q \uparrow} a_{-q \downarrow}\right\rangle\right. \\
& \left.+C_{1 y}\left\langle b_{q \uparrow} b_{-q \downarrow}\right\rangle+C_{1} \psi_{q}\left(\left\langle a_{q \uparrow} b_{-q \downarrow}\right\rangle+\left\langle b_{q \uparrow} a_{-q \downarrow}\right\rangle\right)\right\} K_{q}^{-1} \\
& \quad-\frac{1}{N} \sum_{q}\left\{V_{2}\left(C_{1} \cos k_{y}-C_{2} \gamma_{2 k}\right) \cos q_{y}\right. \\
& \left.+V_{2}^{\prime}\left(-\frac{3}{8}+C_{1} \cos k_{x}-\frac{C_{3}}{2} \cos 2 k_{x}\right) \cos q_{x}\right\} K_{q}^{-1}\left\langle a_{q \uparrow} a_{-q \downarrow}\right\rangle \\
& -\frac{1}{N} \sum_{q}\left\{V_{2}\left(C_{1} \cos k_{x}-C_{2} \gamma_{2 k}\right) \cos q_{x}\right. \\
& \left.+V_{2}^{\prime}\left(-\frac{3}{8}+C_{1} \cos k_{y}-\frac{C_{3}}{2} \cos 2 k_{y}\right) \cos q_{y}\right\} K_{q}^{-1}\left\langle b_{q \uparrow} b_{-q \downarrow}\right\rangle .
\end{aligned}
$$

Here $C_{1 x(1 y)}=C_{1} \sin ^{2} \frac{q_{x(y)}}{2}, \psi_{k}=\sin \frac{k_{x}}{2} \sin \frac{k_{y}}{2}$ and $I_{k}=4 I \gamma_{1 k}$.

\section{EQUATIONS FOR THE SUPERCONDUCTING ORDER PARAMETERS}

For the analysis of the conditions for the appearance of the Cooper instability, we express the anomalous Green's functions in terms of the $\Delta_{l k}^{*}$ parameters in the linear approximation

$$
F_{n m}(k, \omega)=\sum_{l=1}^{3} S_{n m}^{(l)}(k, \omega) \Delta_{l k}^{*} / \operatorname{Det}_{k}(\omega),
$$

where

$$
\begin{aligned}
& \operatorname{Det}_{k}(\omega)=-\operatorname{det}_{k}(\omega) \operatorname{det}_{k}(-\omega), \\
& S_{11}^{(1)}(k, \omega)=Q_{3 y}(k, \omega) Q_{3 y}(k,-\omega), \\
& S_{11}^{(2)}(k, \omega)=S_{22}^{(1)}(k, \omega)=Q_{3}(k, \omega) Q_{3}(k,-\omega), \\
& S_{33}^{(1)}(k, \omega)=K_{k} S_{11}^{(3)}(k, \omega)=K_{k}^{2} Q_{y}(k, \omega) Q_{y}(k,-\omega), \\
& S_{22}^{(2)}(k, \omega)=Q_{3 x}(k, \omega) Q_{3 x}(k,-\omega), \\
& S_{33}^{(2)}(k, \omega)=K_{k} S_{22}^{(3)}(k, \omega)=K_{k}^{2} Q_{x}(k, \omega) Q_{x}(k,-\omega), \\
& S_{12}^{(1)}(k, \omega)=S_{21}^{(1)}(k,-\omega)=Q_{3}(k, \omega) Q_{3 y}(k,-\omega), \\
& S_{12}^{(2)}(k, \omega)=S_{21}^{(2)}(k,-\omega)=Q_{3}(k, \omega) Q_{3 x}(k,-\omega), \\
& S_{12}^{(3)}(k, \omega)=S_{21}^{(3)}(k,-\omega)=K_{k} Q_{x}(k, \omega) Q_{y}(k,-\omega), \\
& S_{33}^{(3)}(k, \omega)=K_{k} Q_{x y}(k, \omega) Q_{x y}(k,-\omega) .
\end{aligned}
$$

The functions used here are

$$
\begin{aligned}
& Q_{x(y)}(k, \omega)=\left(\omega-\xi_{x(y)}\right) J_{y(x)}+t_{k} J_{x(y)}, \\
& Q_{3}(k, \omega)=\left(\omega-\xi_{L}\right) t_{k}+J_{x} J_{y} K_{k}, \\
& Q_{3 x(3 y)}(k, \omega)=\left(\omega-\xi_{L}\right)\left(\omega-\xi_{x(y)}\right)-J_{x(y)}^{2} K_{k}, \\
& Q_{x y}(k, \omega)=\left(\omega-\xi_{x}\right)\left(\omega-\xi_{y}\right)-t_{k}^{2} .
\end{aligned}
$$




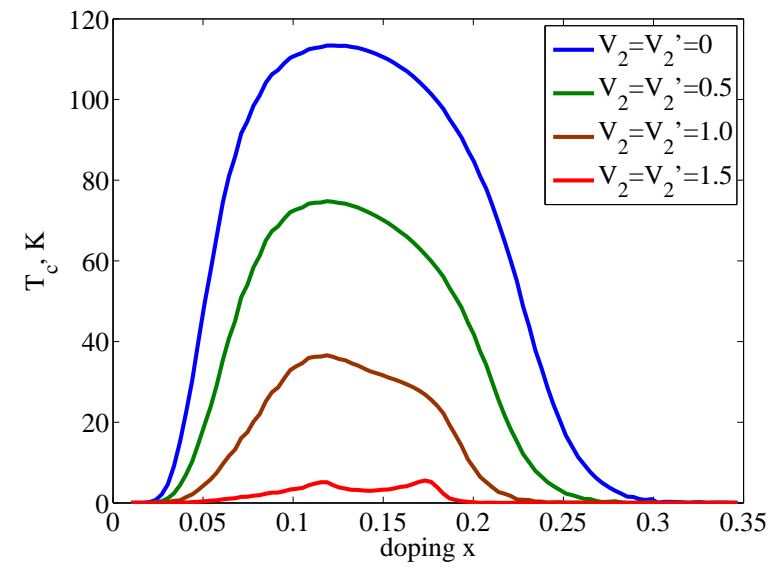

Fig. 2. Critical temperature for the transition to the superconducting $d_{x^{2}-y^{2}}$ phase versus doping at four values of the Coulomb repulsion parameter $V_{2}$ and $V_{2}^{\prime}$.

Using the spectral theorem 23, we find the expressions for the anomalous averages and finally arrive at the closed set of uniform integral equations for the superconducting order parameters $(l=1,2,3)$

$\Delta_{1 k}^{*}=\frac{2}{N} \sum_{l q}\left(V_{2} \cos \left(k_{y}-q_{y}\right)+V_{2}^{\prime} \cos \left(k_{x}-q_{x}\right)\right) M_{11}^{(l)}(q) \Delta_{l q}^{*}$ $\Delta_{2 k}^{*}=\frac{2}{N} \sum_{l q}\left(V_{2} \cos \left(k_{x}-q_{x}\right)+V_{2}^{\prime} \cos \left(k_{y}-q_{y}\right)\right) M_{22}^{(l)}(q) \Delta_{l q}^{*}$, $\Delta_{3 k}^{*}=\frac{1}{N} \sum_{l q}\left\{I_{k-q}\left[C_{1 x} M_{11}^{(l)}(q)+C_{1 y} M_{22}^{(l)}(q)-M_{33}^{(l)}(q)\right.\right.$ $\left.+C_{1} \phi_{q}\left(M_{12}^{(l)}(q)+M_{21}^{(l)}(q)\right)\right]$ $+\left[V_{2}\left(C_{1} \cos k_{y}-C_{2} \gamma_{2 k}\right) \cos q_{y}\right.$ $\left.+V_{2}^{\prime}\left(-\frac{3}{8}+C_{1} \cos k_{x}-\frac{C_{3}}{2} \cos 2 k_{x}\right) \cos q_{x}\right] M_{11}^{(l)}(q)$ $+\left[V_{2}\left(C_{1} \cos k_{x}-C_{2} \gamma_{2 k}\right) \cos q_{x}\right.$ $\left.\left.+V_{2}^{\prime}\left(-\frac{3}{8}+C_{1} \cos k_{y}-\frac{C_{3}}{2} \cos 2 k_{y}\right) \cos q_{y}\right] M_{22}^{(l)}(q)\right\} \frac{\Delta_{l q}^{*}}{K_{q}}$,

where

$$
M_{n m}^{(l)}(q)=\frac{S_{n m}^{(l)}\left(q, E_{1 q}\right)+S_{n m}^{(l)}\left(q,-E_{1 q}\right)}{4 E_{1 q}\left(E_{1 q}^{2}-E_{2 q}^{2}\right)\left(E_{1 q}^{2}-E_{3 q}^{2}\right)} \tanh \left(\frac{E_{1 q}}{2 T}\right) .
$$

Below, we use the system (14) to find the critical superconducting temperature.

In the Fig. 2, we illustrate the results obtained by solving Eq. 14 for the $d_{x^{2}-y^{2}}$-wave pairing, where

$\Delta_{l k}=\Delta_{l 1} \cdot\left(\cos k_{x}-\cos k_{y}\right)+\Delta_{l 2} \cdot\left(\cos 2 k_{x}-\cos 2 k_{y}\right)$.
One can see from Fig. 2 that an increase in $V_{2}$ and $V_{2}^{\prime}$ leads to suppression of the $d$-wave pairing, however superconductivity is maintained up to unphysically large values $V_{2}=V_{2}^{\prime}=1.5 \mathrm{eV}$ of the Coulomb interaction between holes located at the next-nearest-neighbor oxygen ions (for comparison, the intensity of the Coulomb interaction between nearest-neighbor oxygen ions $V_{1}=1-2$ $\mathrm{eV}[16]$ ).

\section{CONCLUSION}

To conclude, we have shown that the intersite Coulomb repulsion between holes located at the nextnearest-neighbor oxygen ions of $\mathrm{CuO}_{2}$ plane suppresses

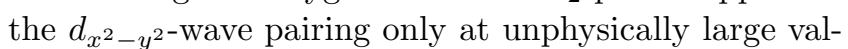
ues of the Coulomb interaction $V_{2}=V_{2}^{\prime}=1.5 \mathrm{eV}$. Taking into account our previous result [11] on cancelation of the effect of the Coulomb interaction $V_{1}$ for the nearest-neighbor oxygen sites on the $d$-wave pairing, we conclude that an account for the real structure of $\mathrm{CuO}_{2}$ plane leads to stability of the $d_{x^{2}-y^{2} \text {-wave pair- }}$ ing towards the strong intersite Coulomb repulsion. It is obvious that an account for the Coulomb interaction $V_{3}$ does not effect on the superconducting $d$-wave pairing because of the same "symmetry reason" as that for $V_{1}[1]$.

The work was supported by the Russian Foundation for Basic Research (RFBR) and partly by the Government of Krasnoyarsk Region (project nos. 16-42240435 and 16-42-243057). The work of A.F.B. was supported by RFBR (project no. 16-02-00304). The work of M.M.K. was supported by grant of the President of the Russian Federation (project MK-1398.2017.2).

1. V. J. Emery, Phys. Rev. Lett. 58 (1987), p. 2794.

2. C. M. Varma, S. Schmitt-Rink and E. Abrahams, Solid State Commun. 62 (1987), p. 681.

3. R. O. Zaitsev and V. A. Ivanov, Sov. Phys. Solid State 29 (1987), p. 1475.

4. Yu. A. Izyumov, Phys. Usp. 40 (1997), p. 445; 42 (1999), p. 215.

5. N. M. Plakida, High-Temperature Cuprate Superconductors, Springer-Verlag, Berlin-Heidelberg (2010).

6. R. O. Zaitsev, JETP 98 (2004), p. 780.

7. V.V. Val'kov and M. M. Korovushkin, JETP 112 (2011), p. 108.

8. V.Yu. Yushankhai, G. M. Vujicic and R. B. Zakula, Phys. Lett. A 151 (1990), p. 254.

9. V. V. Val'kov, T. A. Val'kova, D. M. Dzebisashvili and S. G. Ovchinnikov, JETP Lett. 75 (2002), p. 378.

10. N. M. Plakida and V.S. Oudovenko, Eur. Phys. J. B 86 (2013), p. 115; JETP 146 (2014), p. 631. 
11. V.V. Val'kov, D. M. Dzebisashvili, M. M. Korovushkin and A. F. Barabanov, JETP Lett. 103 (2016), p. 385.

12. N. M. Plakida, arXiv: 1607.02935.

13. A.F. Barabanov, L.A. Maksimov and G. V. Uimin, JETP Lett. 47 (1988), p. 622.

14. J. Zaanen and A. M. Oleś, Phys. Rev. B. 37 (1988), p. 9423.

15. M. S. Hybertsen, M. Schluter and N.E. Christensen, Phys. Rev. B 39 (1989), p. 9028.

16. M. H. Fischer and E.-A. Kim, Phys. Rev. B 84 (2011), p. 144502.

17. M. Ogata and H. Fukuyama, Rep. Prog. Phys. 71 (2008), p. 036501.

18. D. M. Dzebisashvili, V. V. Val'kov and A.F. Barabanov, JETP Lett. 98 (2013), p. 528.

19. V. V. Val'kov, D. M. Dzebisashvili and A.F. Barabanov, JETP 118 (2014), p. 959.

20. R. Zwanzig, Phys. Rev. 124 (1961), p. 983.

21. H. Mori, Prog. Theor. Phys. 33 (1965), p. 423.

22. V. V. Val'kov, D. M. Dzebisashvili and A. F. Barabanov, Phys. Lett. A 379 (2015), p. 421.

23. D. N. Zubarev, Sov. Phys. Usp. 3 (1960), p. 320. 